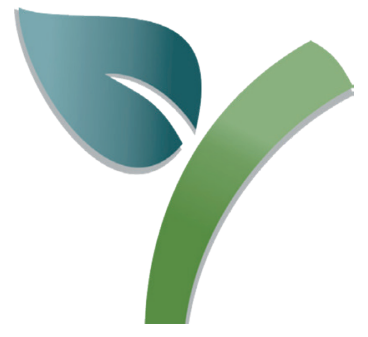

\title{
ÓLEOS ESSENCIAIS NO TRATAMENTO DE SEMENTES
}

\author{
Daniele Maria do Nascimento ${ }^{1}$, Marcos Roberto Ribeiro-Junior ${ }^{1}$, \\ Paula Leite dos Santos ${ }^{1}$, Alana Emanoele Pereira ${ }^{1}$, Adriana Zanin Kronka ${ }^{1}$
}

\section{RESUMO}

O interesse pelo controle alternativo de doenças de plantas tem crescido nos últimos anos, em parte porque os consumidores estão cada vez mais exigentes em relação aos alimentos que consomem, mas, principalmente, devido à resistência que vários fitopatógenos têm desenvolvido aos principais defensivos empregados. Diante desse contexto, os óleos essenciais vêm ganhando destaque. Na área de patologia de sementes, os estudos realizados em nosso país têm focado na inibição in vitro de patógenos transmitidos por sementes por óleos essenciais, e na aplicabilidade dessas substâncias às sementes, avaliando-se a qualidade fisiológica. No entanto, em outros países, pesquisadores vêm solucionando um dos maiores problemas relacionados ao tratamento de sementes com óleos essenciais: a volatilização. O encapsulamento dessas substâncias permite que sejam usadas a campo, sem perder efeito ao longo do tempo, viabilizando, assim, seu uso em tratamento de sementes. A presente revisão bibliográfica visa abordar todos esses aspectos, auxiliando assim pesquisadores da área.

PALAVRAS-CHAVE: Controle alternativo, Patologia de sementes, Patógenos de sementes, Produtos naturais, Tratamento alternativo de sementes.

ESSENTIAL OILS IN SEED TREATMENT

\section{ABSTRACT}

Interest in alternative control of plant diseases has grown in recent years, not only because consumers have increasingly been more demanding concerning the food they consume, but also mainly due to the resistance that various phytopathogens have developed to the main pesticides used. In this context, essential oils have been standing out in the market. In seed pathology area, studies carried out in our country have focused on the in vitro inhibition of seedborne pathogens by essential oils, and on the applicability of these substances to seeds, evaluating the physiological quality. However, in other countries, researchers have been solving one of the biggest problems related to the seeds treatment with essential oils: volatilization. Those substances encapsulation allows them to be used in the field, without decreasing their effectiveness over time, thus enabling their use in seed treatment. This review aims to address aspects related to essential oils and seed treatment, providing subsidies for greater employability of this alternative plant disease control technique. KEYWORDS: Alternative control, Seed pathology, Seed pathogens, Natural products, Alternative seed treatment. 


\section{INTRODUÇÃO}

Antigamente, extensas áreas eram cultivadas com plantas para fins de controle fitossanitário. Muitas espécies de plantas fornecem ingredientes ativos que são usados até hoje como fungicidas e inseticidas, como é o caso de Derris spp. e Lonchocarpus spp. (rotenona), crisântemos (piretro) e fumo (nicotina). Com o avanço da Segunda Guerra Mundial, essas áreas foram dizimadas, iniciando assim o desenvolvimento dos produtos sintéticos. Inicialmente, essa parecia ser a solução no controle de pragas e doenças na agricultura mundial (MORAIS 2009a).

O uso contínuo de algumas classes de defensivos sintéticos resultou na seleção de microrganismos resistentes aos princípios ativos. $O$ agente causal da ferrugem da soja, Phakopsora pachyrhizi, por exemplo, é comprovadamente resistente a alguns ingredientes ativos empregados em formulações fungicidas (CHECHI et al. 2020; GHINI \& KIMATI 2000).

Além disso, os consumidores anseiam cada vez mais por alimentos saudáveis, produzidos de modo sustentável e portadores de selos que certificam a não utilização de agrotóxicos. Para tanto, o mercado precisa suprir essa nova demanda, e os agricultores orgânicos necessitam de produtos naturais que possam ser empregados no controle de pragas e doenças.

As doenças causadas por patógenos transmitidos via sementes representam um desafio na produção orgânica, onde os produtos disponíveis para tratamento de sementes são ainda mais limitados (RICCIONI et al. 2019). O uso de sementes sadias é uma das principais medidas a ser adotada no manejo de doenças, reduzindo a disseminação e introdução do patógeno em novas áreas, e protegendo a semente de eventuais infecções que possam vir a causar damping off ou redução na produtividade.

Na última década, muitas pesquisas focaram nos produtos naturais oriundos de plantas, como por exemplo, extratos vegetais e óleos essenciais (OEs), que possuem propriedades antimicrobianas comprovadas. A possível substituição dos químicos por alternativas mais sustentáveis tem impulsionado essa área de estudo, com aumento significativo de trabalhos nos últimos anos.

Os OEs podem ser extraídos de várias partes de plantas aromáticas. São misturas complexas de voláteis e semivoláteis, odoríferos, solúveis em solventes orgânicos e insolúveis em água (BASAK \& GUHA 2017; ZUZARTE; SALGUEIRO 2015). Os principais componentes dos OEs são terpenos de hidrocarbonetos (monoterpenos, sesquiterpenos e diterpenos) e terpenos oxigenados (ésteres, aldeídos, cetonas, álcoois, fenóis e epóxidos) (KAYODE \& AFOLAYAN 2015).

O emprego de OEs no controle de doenças já está bem descrito na literatura e o fato de serem biodegradáveis, não persistindo no solo e na água, e de fácil extração, tem encorajado seu uso na agricultura (ARSHAD et al. 2014; ISMAN 2000; ISMAN \& MACHIAL 2006; MISRA \& PAVLOSTATHIS 1997; STURCHIO et al. 2014; ZYGADLO \& GROW 1995).

Siddiqui et al. (2017) pontuam que os OEs podem representar uma nova classe de defensivos, mais seguros para o meio ambiente e biodegradáveis. No entanto, sua natureza instável, devido a alta volatilidade, rápida degradação e baixa miscibilidade, pode dificultar seu uso, especialmente, em formulações para tratamento de sementes.

Morais (2009a) abordou os principais estudos já conduzidos com OEs em nosso país, com resultados relevantes para o manejo integrado de doenças. Na patologia de sementes, poucos são os pesquisadores dedicados à pesquisa com OEs. Infelizmente, não vêm sendo realizados avanços significativos nessa área. Os estudos têm se limitado à descoberta de quais OEs podem ser empregados em tratamento de sementes, obtendo bons índices no controle de patógenos, sem afetar, contudo, a germinação. Trabalhos dessa natureza são importantes e são também o primeiro passo no desenvolvimento de novas formulações de produtos.

Os trabalhos advindos de outros países evidenciam a importância da busca por métodos alternativos no tratamento de sementes, uma vez que vários microrganismos de interesse agronômico vêm apresentando resistência aos principais produtos usados, entre outros motivos já discutidos acima. Apesar de já haver produtos desenvolvidos à base de OEs em outros países, no Brasil, seu uso na agricultura ainda é escasso, e apenas o óleo de neem vem sendo empregado em algumas formulações.

A presente revisão bibliográfica visa abordar os principais aspectos referentes ao tratamento de sementes com OEs, como: o controle de fitopatógenos, os possíveis danos às sementes e os mais recentes avanços na formulação desses OEs. Com 
isso, espera-se incentivar o envolvimento de mais pesquisadores nessa área e auxiliar no desenvolvimento de novos estudos.

\section{CONTROLE DE FITOPATÓGENOS EM SEMENTES POR ÓLEOS ESSENCIAIS}

Para serem considerados eficientes no tratamento de sementes visando ao controle de fitopatógenos, os OEs (ou seus compostos), devem, além de controlar eficientemente o patógeno alvo, não apresentar efeitos adversos à germinação das sementes e ao desenvolvimento das plântulas. Neste tópico estão descritos alguns exemplos de estudos em que os OEs podem ser recomendados para o tratamento de sementes infectadas e/ou contaminadas.

Em sementes de soja, o controle de Colletotrichum truncatum pelos OEs de hortelã do campo (Hyptis marrubioides), alfazema-do-Brasil (Aloysia gratissima) e erva-baleeira (Cordia verbenacea) foi superior ao tratamento fungicida com carbendazim (SILVA et al. 2012). Os OEs de copaíba (Copaifera langsdorfii) e manjericão (Ocimum basilicum) reduziram a incidência de fungos associados às sementes de feijão-fava, como espécies de Aspergillus spp., Cladosporium sp., Fusarium spp. e Penicillium spp. (GOMES et al. 2016).

Sementes de feijão-de-corda ou caupi (Vigna unguiculata), tratadas nas concentrações de 0,5\% e $1 \%$ do OE de pimenta-de-macaco (Piper aduncum), apresentaram menor incidência de fungos, em especial, Macrophomina phaseolina (LOBATO et al. 2007). As sementes foram tratadas pela técnica de imersão, durante cinco minutos. Dias et al. (2019) também relataram controle deste patógeno em sementes de feijão-caupi, mas dessa vez pelo $O E$ de alecrim pimenta (Lippia sidoides). A concentração de $4 \mathrm{~mL} / \mathrm{kg}$ de semente também inibiu demais patógenos associadas a semente, como Alternaria sp, Aspergilus sp., Cladosporium, Fusarium spp, Penicillium sp., Phoma sp. e Rhizopus, e não afetou o poder germinativos das sementes. Hillen et al. (2012) verificaram que os OEs de candeia e palmarosa inibiram o crescimento de Rhizopus sp., Aspergillus sp. e Penicillium sp. em sementes de feijão.

Os OEs de cravo, tea tree e tomilho (Thymus vulgaris), reduziram o percentual de infecção de sementes de ervilha por fungos do gênero Didymella (RICCIONI et al. 2019). Os autores ainda avaliaram dois métodos de aplicação, por submersão e spray. Apesar dos dois métodos controlarem eficientemen- te o fungo, o método por spray apresentou algumas vantagens, como menor quantidade de ingrediente ativo aplicado e, consequentemente, menor custo. Para o tratamento por spray, foram necessários $50 \mathrm{~mL}$ da emulsão de OE para cada kg de semente, contra 1 litro para a submersão das sementes. Dependendo do OE, o custo com o tratamento por submersão pode ser até treze vezes maior.

Nguefack et al. (2008) testaram os efeitos dos OEs de capim-limão, manjericão e tomilho em sementes de arroz infectadas por Alternaria padwickii, Bipolaris oryzae e Fusarium moniliforme. Os autores relataram que os tratamentos com os OEs controlaram a infecção das sementes, reduzindo ainda a transmissão dos fungos para plântulas e com um acréscimo na germinação de 5 a 13\%, se comparado com as sementes não tratadas.

A incidência de patógenos dos gêneros Penicillium e Fusarium foi reduzida em sementes de miIho tratadas com OEs de eucalipto (Corymbia citriodora e Eucalyptus calmadulensis, respectivamente), resultando em controle destes superiores ao obtido com fungicida. Os tratamentos consistiram na imersão das sementes (850 g) em $500 \mathrm{~mL}$ de acetona + 1,7 mL de cada OE (DOMENE et al. 2016). Comumente, em estudos da área, é empregado o Tween como agente emulsionante, a fim de facilitar a dispersão dos OEs, mas, neste caso, através de testes preliminares, os autores constataram que a acetona era mais eficiente nesse aspecto. Quando usada isoladamente no tratamento das sementes, a acetona apresentou pouca ação fungicida, mas não se pode descartar um possível efeito aditivo da mistura do solvente com o OE.

De acordo com Rodrigues et al. (2006), a fumigação do OE de alfavaca-cravo (Ocimum gratissimum) em sementes de trigo naturalmente infectadas por Bipolaris sorokiniana se mostrou uma alternativa promissora ao tratamento químico. Em torno de 100 sementes foram armazenadas em saquinhos de gaze, colocados dentro de frascos fechados, contendo um recipiente com $2 \mathrm{~mL}$ do OE. As sementes foram expostas ao OE por diferentes períodos (7, 14, 21 e 28 dias), e os autores relataram um aumento na incidência de sementes infectadas após sete dias de exposição, possivelmente devido à volatilização, sendo este considerado o período ideal de exposição.

Sementes de pimentão infectadas por Colletotrichum gloeosporioides e sementes de feijão 
infectadas por $M$. phaseolina tratadas com o $\mathrm{OE}$ de cravo, através da submersão em água destilada com OE na concentração de $0,25 \%$ emulsionado em Tween 80, apresentaram menor índice de incidência dos referidos patógenos (NASCIMENTO et al. 2020; SANTOS 2018). Para sementes de erva-doce e mandacaru, o OE de anis (Pimpinella anisum), além de promover um acréscimo na germinação, controlou eficientemente fungos que infestavam as sementes (MATA et al. 2009; ARAÚJO NETO et al. 2012).

Os OEs de alecrim (Rosmarinus officinalis) e eucalipto (Eucalyptus globulus), na concentração de $2 \%$, demonstraram ser promissores para o tratamento de sementes de tomate, no manejo da mancha bacteriana, causada por Xanthomonas spp. (MBEGA et al. 2012). Espécies desse gênero bacteriano já foram descritas como resistentes ao cobre na Tanzânia, o que motivou a pesquisa por tratamentos alternativos nesse país (SHENGE 2006). Ohno et al. (2003) consideram baixos os riscos de bactérias adquirirem resistência aos OEs e seus compostos.

A imersão de sementes por 30 minutos nos OEs de canela, cravo e tomilho eliminou $99 \%$ das bactérias associadas a semente de repolho, entre elas, Clavibacter michiganensis e Xanthomonas campestris pv. campestris (VAN DER WOLF et al. 2008). O eugenol é capaz de reduzir a população bacteriana de $X$. campestris pv. phaseoli, até na concentração de $1 \mathrm{mg} / \mathrm{mL}$, em sementes de feijão (LO CANTORE et al. 2009).

O mercado do controle biológico vem crescendo no país, ao contrário dos OEs que, com exceção do óleo de neem e do extrato de folhas de tea tree, não possuem produtos registrados no $\mathrm{Mi}$ nistério da Agricultura, Pecuária e Abastecimento (MAPA).

Estudos mostraram que quando usados em associação ao Trichoderma viride ou Bacillus subtilis, os OEs não causam danos a esses microrganismos ou reduzem a sua eficiência. O OE de tomilho, em associação $T$. viridae ou $B$. subtilis, em tratamento de sementes de melão, pepino, pimenta e tomate, controlou eficientemente a incidência de podridão radicular causada por diversos patógenos (Alternaria solani, F. solani, F. oxysporum, R. solani, Sclerotium rolfsii, $M$. phaseolina e Pythium sp.). Os resultados obtidos foram superiores aos dos tratamentos somente com o OE ou com os agentes de biocontrole isolados (EL-MOUGY et al. 2012).

De modo semelhante, o tratamento de se- mentes de feijão com os OEs de cominho (Carum carvi), cravo (Dianthus caryophyllus), gerânio (Geranium viscosissimum), menta (Mentha piperita) ou tomilho, e a posterior semeadura em solo tratado com $T$. harzianum, resultou na redução de incidência de podridão-radicular (F. solani, R. solani, S. rolfsii e M. phaseolina) (ABDEL-KADER et al. 2011).

\section{ÓLEOS ESSENCIAIS EM SEMENTES E GRÃOS ARMAZENADOS E IN NATURA}

A contaminação de produtos alimentícios por fungos toxigênicos gera graves problemas relacionados à segurança alimentar. Aspergillus flavus é uma das principais espécies fúngicas responsáveis pela deterioração de alimentos, causando danos severos a várias commodities agrícolas antes da pré-colheita e após a colheita (LI et al. 2020). Outro fator de risco associado às espécies de Aspergillus é a produção de aflatoxinas, metabólitos secundários altamente tóxicos a humanos e animais (ABDIN et al. 2010; PERAICA et al. 1999). Estratégias para controlar o crescimento dessas espécies fúngicas em grãos armazenados são necessárias e os OEs podem contribuir nesse sentido.

A fumigação do $\mathrm{OE}$ de gengibre em sementes de milho, nas concentrações de 25 e 50 ug/g, inibiu a produção de aflatoxina e o crescimento fúngico de A. flavus (NERILO et al. 2020). Outros OEs também já foram recomendados para o tratamento de sementes de milho, com a mesma finalidade. Os OEs de canela, cravo, erva-de-santa-maria (Teloxys ambrosioides), manjericão (Ocimum basilicum), menta (Mentha piperita), orégano (Origanum vulgare) e tomilho inibiram totalmente o desenvolvimento de A. flavus nos grãos. Nesse caso, as sementes foram imersas no OE puro por 30 minutos, e o tratamento proporcionou um efeito residual por até quatro semanas (MONTES-BELMONT \& CARVAJAL 1998). Segundo Nerilo et al. (2020), os OEs permanecem bioativos como vapores, viabilizando assim a proteção de grãos armazenados.

Em sementes de lótus infectadas com $A$. flavus e tratadas com OE de anis estrelado, o crescimento fúngico, assim como a produção de aflatoxina B1 (AFB1) e aflatoxina B2 (AFB2), foram completamente inibidos na concentração de 6,0 uL/g (LI et al. 2020). As sementes de lótus são consumidas in natura e altamente suscetíveis à contaminação microbiana (SINDHU et al. 2011). O uso desse OE aumentou consideravelmente o tempo de prateleira, 
sem apresentar efeitos adversos ao consumidor (LI et al. 2020).

Em outro estudo, Bankole et al. (2005) demonstraram que o $\mathrm{OE}$ de capim-limão, na concentração de 0,5 a $1 \mathrm{~mL}$ a cada $100 \mathrm{~g}$ de sementes de melão, reduziu significativamente a deterioração e produção de aflatoxinas por $A$. flavus.

Kumar (2016) analisou a microbiota de sementes de ervilhas obtidas em mercados, e verificaram a presença de ao menos 15 espécies de fungos, com destaque para espécies de Aspergillus (A. flavus, A. niger e A. ochraceous). O tratamento com o OE de cominho (Cuminum cyminum), além de controlar eficientemente todos os patógenos por até 120 dias em armazenamento, sendo superior até mesmo ao expurgo com fosfina, não alterou as propriedades organolépticas das sementes. As sementes foram armazenadas em sacos plásticos de polietileno, contendo aproximadamente $400 \mathrm{~g}$ de sementes, e o OE foi adicionado nas doses de 0,50 $\mathrm{mL}$ (1.000 ppm) e 0,76 mL (1.500 ppm).

\section{MECANISMOS DE AÇÃO DE ÓLEOS ESSENCIAIS SOBRE FITOPATÓGENOS}

O mecanismo exato de ação dos OEs sobre fitopatógenos ainda é desconhecido, embora vários estudos venham tentando elucidar essa questão. Interessantemente, um mesmo OE pode causar danos irreversíveis a um patógeno, mas não afetar outros. É o caso do OE de cravo (Syzygium aromaticum), que em estudo realizado por Costa et al. (2011), apesar de ter causado alterações morfológicas em Rhizoctonia solani, Fusarium solani e Fusarium oxysporum, como desorganização dos conteúdos celulares, ocorrência de vacúolos, diminuição da nitidez da parede celular, entre outros, não causou danos a Macrophomina phaseolina. Esse último foi capaz de retomar seu crescimento após exposição ao OE. No referido estudo, o OE continha $83 \%$ de eugenol em sua composição, um composto fenólico já bem documentado por sua ação fungicida (CHENG et al. 2008; RANA et al. 2011)

Segundo Wang et al. (2010), o eugenol pode se ligar à membrana fúngica, alterando sua permeabilidade e levando-a ao rompimento. Deve-se ressaltar ainda que esse composto, apesar de inibir o crescimento micelial de Botrytis cinerea, não afetou a germinação de conídios, indicando que sua atuação talvez esteja voltada para os estágios pós-germinação. De modo semelhante, fungicidas como o carbendazim, por exemplo, têm pouco ou nenhum efeito na germinação de esporos, mas inibem o crescimento micelial (SHERALD et al. 1973; SUZUKI et al. 1984). A germinação de conídios de C. gloeosporioides, por sua vez, foi totalmente inibida pelos OEs de capim-limão (Cymbopogon citratus), cravo, eucalipto citriodora (Corymbia citriodora), gengibre (Zingiber officinale), gerânio (Pelargonium hortorum), pimenta-preta (Piper nigrum), menta (Mentha arvensis) e tea tree (Melaleuca alternifolia) (NASCIMENTO et al. 2019).

O geraniol, composto majoritário de alguns OEs, provocou o vazamento de potássio das células fúngicas. Estudos demonstraram, ainda, que em células bacterianas também ocorreram vazamentos de íons de potássio e aumento de permeabilidade do citoplasma, devido ao contato com o OE de tea tree (BARD et al. 1988; COX et al. 2000).

O ergosterol é o principal componente da membrana celular fúngica e também alvo dos fungicidas químicos que interrompem as vias envolvidas em sua biossíntese. Na concentração de 3,6 $\mu \mathrm{L} /$ $\mathrm{mL}$, o OE de anis estrelado (Illicium verum) é capaz de inibir completamente a produção de ergosterol de $A$. flavus (LI et al. 2020). Ademais, por meio de microscopia eletrônica de varredura (MEV), foram observadas alterações na morfologia de hifas, conidióforos e esporos de $A$. flavus, decorrentes da ação desse OE.

Manso et al. (2013) também evidenciaram os danos decorrentes da exposição de $A$. flavus ao $\mathrm{OE}$ de canela (Cinnamomum zeylanicum). Na concentração de $10 \mathrm{~mL}$ do $\mathrm{OE}$, ocorreu o rompimento de hifas e os poucos conidióforos observados apresentaram conídios incompletos. Com $20 \mathrm{~mL}$, apenas esporos individuais, porém deformados, foram observados em MEV e, em concentrações superiores a $20 \mathrm{~mL}$, ocorreu total inibição da esporulação.

Em relação aos patógenos bacterianos, os efeitos decorrentes da exposição ao OE podem ser bacteriostáticos, quando inibem o crescimento, ou bactericidas, quando ocorre a morte celular. Além do mais, bactérias Gram-positivas e Gram-negativas reagem de modo diferente aos OEs (SWAMY et al. 2016). Os OEs de sândalo (Santalum album) e capim-vetiver (Chrysopogon zizanioides) inibiram bactérias Gram-positivas, mas não Gram-negativas (HAMMER \& CARSON 2011; RAUT \& KARUPPAYIL 2014).

A inibição do quorum-sensing (QS) por OEs 
também foi relatada por Szabó et al. (2010). O papel do QS na patogenicidade de fitobactérias já é bem conhecido e uma das consequências de sua inibição é a redução da patogenicidade e da resistência de bactérias aos antibióticos.

Os terpenos afetam diferentes funções metabólicas vitais, como a respiração, e interferem na membrana citoplasmática de bactérias Gram-negativas, ocasionando sua desintegração (COX et al. 1998; TASSOU et al. 2000; WALSH et al. 2003).

\section{COMPOSIÇÃO QUÍMICA DOS ÓLEOS ESSENCIAIS}

Óleos essenciais extraídos de uma mesma espécie vegetal podem variar quimicamente entre si, devido a fatores genéticos, como a cultivar, e ambientais, tais como, localização geográfica, época de coleta e condições durante o processo de extração. A parte da planta selecionada para extração dos OEs (caule, folhas, raízes ou frutos) também é um fator importante na composição química destes.

Um exemplo é aroeira-salsa (Schinus molle). O OE extraído do fruto é rico em $\alpha$-phellandrene $(31 \%), \beta$-phellandrene (16\%) e limonene (16\%), não apresentando índices satisfatórios no controle de bactérias, provavelmente devido à alta quantidade de monoterpenos (ERYIGIT et al. 2017). Óleos essenciais extraídos de ramos e folhas, por sua vez, apresentaram uma composição química diferente. O OE extraído do ramo registrou maior quantidade de $\delta$-cadieno $(2,77 \%)$, viridifloral $(21,74 \%)$, copaeno $(12,9 \%)$ e cariofileno $(9,99 \%)$, enquanto que, quando extraído das folhas, os compostos majoritários foram germacreno D (39\%), a-pineno $(14,77 \%)$ e germacreno $B(6,25 \%)$ (MENEZES FILHO \& SOUZA 2020a). O percentual de controle de fitopatógenos também pode variar. Nesse caso, na concentração de $100 \mu \mathrm{L}$, o crescimento micelial de Sclerotinia sclerotiorum foi inibido em $86 \%$ e $81 \%$ pelo uso do OE dos ramos e das folhas, respectivamente.

O perfil químico dos OEs de guamirim (Myrcia guianensis), espécie típica do Cerrado, quando extraídos da flor e do fruto, também diferem entre si. No OE da flor, os compostos majoritários foram metil eugenol $(20,27 \%)$, geraniol $(7 \%)$ e valenceno $(6,75 \%)$. E silvestreno (11,57\%), valeceno $(10,63 \%)$ e acetato de farnesila (20,29\%), para o OE do fruto. Cepas de $S$. sclerotiorum e $C$. gloeosporioides foram altamente sensiveis ao OE do fruto, com índices de inibição do crescimento próximas a $100 \%$. Mas, para o OE da flor, C. gloeosporioides apresentou maior resistência e S. sclerotiorum, assim como A. flavus, que não havia sido inibido pelo $O E$ do fruto, apresentaram $100 \%$ de inibição do crescimento (MENEZES FILHO et al. 2020b).

As estações do ano também influenciam na composição e rendimento dos OEs. O OEs extraídos das folhas da goiaba durante os períodos chuvosos apresentaram maior rendimento se comparado ao período seco. Não só a composição química, como também a concentração relativa de alguns componentes diferiu entre as estações. De modo geral, os compostos majoritários permaneceram os mesmos, $\alpha$-Humulene $(26,37 \%$ na estação chuvosa e 18,74 na estação seca) e trans-Caryophyllene $(18,90 \%$ na estação chuvosa e $13,44 \%$ na estação seca). Mas alguns compostos, embora em menores concentrações, como por exemplo, o monoterpeno 1,8-cineole $(1,50 \%)$, só esteve presente no OE coletado durante o período chuvoso. Quando avaliados no controle de S. sclerotiorum, ambos os OEs foram eficientes, inibindo o crescimento micelial em mais de 93\% (SILVA et al. 2018).

Ribeiro et al. (2018) demonstraram que além da época, o horário da colheita também infere na composição dos OEs de três espécies vegetais do gênero Croton. As 12 horas, houve maior rendimento na extração do OE de $C$. zenhtneri, se comparado as extrações realizadas as 8 e 20 horas. De modo semeIhante, a colheita próxima às $12 \mathrm{~h}$ também proporcionou maior rendimento do OE de $C$. citratus em relação aos demais horários de extração (MORAIS 2009b). Segundo Tais \& Zeiger (2013), altos níveis de radiação solar estimulam maior produção de metabólitos secundários, através de reações biossintéticas que são realizadas na fotossíntese.

Todos esses fatores que influenciam a composição final dos OEs e, consequentemente, a sua eficácia no controle de fitopatógenos devem ser considerados no momento da produção de espécies vegetais para esse fim. A rotulagem adequada dos OEs pelas empresas fabricantes é outro aspecto que merece atenção.

A coleta, extração e identificação dos compostos presentes nos OEs são processos que demandam tempo e recursos dos pesquisadores. Muitos optam pela obtenção de OEs comerciais, com uma produção padronizada e um produto final sem maiores variações. Contudo, a maioria dos OEs disponíveis no mercado não disponibiliza maiores detalhes acerca de sua composição química, inviabili- 
zando toda a pesquisa realizada.

Para que de fato os OEs sejam implementados no controle de doenças, é necessário um esforço por parte de todos os envolvidos. Se por um lado, há um crescente aumento da participação dos OEs no setor de beleza e cuidados pessoais, na agricultura, os OEs caminham a passos lentos, mesmo diante de todos os benefícios já apresentados. A devida rotulagem desses produtos pode vir a contribuir em seu emprego nas lavouras.

\section{ALELOPATIA DE ÓLEOS ESSENCIAIS NA GERMINAÇÃO E DESENVOLVIMENTO DE PLÂNTULAS}

A alelopatia pode ser definida como a interferência benéfica ou prejudicial de uma planta no desenvolvimento de outra, sejam plantas cultivadas ou daninhas, devido à liberação de metabólitos secundários (FRAENKEL 1959; STAMP 2003). Como competem constantemente com outras espécies vegetais por espaço, luz e nutrientes, as plantas dispõem de uma vasta gama desses metabólitos, como os OEs.

Até então, foram ressaltados os benefícios dessas substâncias no controle de fitopatógenos em sementes, mas os malefícios na germinação de sementes também são evidentes, uma vez que OEs são usados no desenvolvimento de herbicidas naturais mais potentes e menos prejudiciais a outros organismos (CHOU 1999; DUKE et al. 2002; VYVYAN 2002). Em muitos países, herbicidas sintéticos regulamentados para a agricultura orgânica são constituídos por OEs e seus componentes (LANINI 2010; YOUNG 2004).

O tratamento de sementes com OEs para fins fitossanitários deve levar em consideração: a) o $\mathrm{OE}$ ou composto selecionado, pois alguns são naturalmente mais fitotóxicos que outros, mas a resposta também pode variar de acordo com a espécie vegetal; b) a semente a ser tratada; c) o tempo de exposição; e d) a técnica de tratamento.

Os OEs são constituídos principalmente por terpenos e seus derivados, que são compostos formados por carbono e hidrogênio (hidrocarbonetos). Quando adicionado um oxigênio à cadeia, são denominados de terpenoides e apresentam diferentes funções químicas, como ácidos, álcoois, aldeídos, cetonas, éteres, fenois ou epóxidos terpênicos (FELIPE \& BICAS 2017).

Segundo Rolli et al. (2014), OEs com alto teor de álcoois monoterpênicos e aldeídos possuem maior atividade fitotóxica. No entanto, enquanto os álcoois monoterpênicos agem tanto nas sementes como em plântulas, os aldeídos interferem principalmente no desenvolvimento de plântulas. A associação desses constituintes no desenvolvimento de herbicidas pode levar ao produto ideal no controle de plantas daninhas, com capacidade de atuar na pré e pós-emergência.

Outros pontos importantes para o tratamento de sementes com OEs são o método de aplicação e período de exposição. Os mais usados são o tratamento por submersão das sementes, que apresenta a vantagem de ser facilmente implementado em nível de fazenda, e a fumigação, em que as sementes permanecem em ambiente fechado e são expostas ao OE, por tempo determinado (ORZALI et al. 2014; SOMDA et al. 2007).

Riccioni et al. (2019) salientam que os estudos devem ser direcionados a cada patossistema específico, testando-se diferentes OEs/compostos e ajustando-se a dose e duração de tratamento, de modo que não ocorram danos adversos aos hospedeiros, e o patógeno seja eficientemente controlado. Na concentração de $0,25 \%$, Santos (2018) obteve uma boa germinação de sementes de feijão tratadas por submersão com o OE de cravo, aliado ao controle de $M$. phaseolina nas sementes. Concentrações superiores do OE foram mais eficientes em controlar o patógeno, porém, afetaram negativamente a germinação e emergência de plântulas.

Para a fumigação, as metodologias são diversas e podem implicar em menor ou maior exposição das sementes e, consequentemente, fitotoxidez. Ajayi et al. (2014) armazenaram $50 \mathrm{~g}$ de sementes de feijão em frascos de $1 \mathrm{~L}$ e, na parte inferior da tampa do frasco, continha um papel de filtro embebido pelo composto estudado. Os quatros compostos (carvacrol, 1,8-cineol, eugenol e mentona), nas doses de 10 e $20 \mu \mathrm{L} / \mathrm{L}$, afetaram negativamente a germinação e crescimento de mudas. No entanto, em sementes armazenadas, a fitotoxicidade tende a diminuir com o tempo, devido à biodegradação dos OEs (AJAYI et al. 2014).

Em outra abordagem, Hillen et al. (2012) distribuíram sementes em camada única em gerbox, sobre dois papeis de filtro umedecidos com água. No interior da gerbox, foi adicionado um recipiente contendo $200 \mu \mathrm{L}$ do OE puro, por 10 minutos. Para as sementes de soja e milho, essa exposição aos OEs de 
alecrim, candeia (Eremanthus erythropappus) e palmarosa (Cymbopogon martini) foi prejudicial à germinação, mas para as sementes de feijão, apenas O OE de alecrim apresentou efeitos adversos. Em relação ao OE de palmarosa, nenhuma das sementes de soja fumigadas germinou, enquanto que nas sementes de feijão e milho, $87 \%$ e $77 \%$ de sementes assintomáticas germinadas, respectivamente, foram verificadas. Esses resultados evidenciam que diferentes espécies vegetais reagem de modo distinto a um mesmo OE. Segundo os autores, três fatores podem ter ocasionado baixa germinação, sendo eles concentração, tempo de exposição e/ou compostos presentes nos OEs. O OE de palmarosa possui um alto teor (entre 70 a $90 \%$ ) de compostos terpenoides, como o limoneno, $\beta$-ocimeno, linalol, geraniol acetato e $\beta$-cariofileno, substâncias comprovadamente alelopáticas, mas que, no referido estudo, não afetaram a germinação das sementes de feijão.

Um dos aspectos negativos do tratamento por submersão é o contato direto das sementes com o $O E$, o que não ocorre nas sementes fumigadas. Segundo Riccioni et al. (2019), a embebição excessiva de sementes de ervilha com OEs pode ter comprometido a germinação através de danos causados ao tegumento. Corroborando com essa hipótese, Van der Wolf et al. (2008) verificaram que períodos de embebição superiores a uma hora ocasionaram danos às sementes de repolho, mas períodos inferiores podem ser recomendados com segurança para desinfecção das sementes. De fato, sementes de pimentão imersas em OEs por cinco minutos apresentaram germinação superior a $85 \%$ (NASCIMENTO et al. 2020). O curto período de exposição das sementes aos OEs pode promover o controle de fitopatógenos sem chegar a causar danos expressivos às sementes.

O eugenol, um dos principais compostos de OEs, é responsável pela diminuição da atividade da $\alpha$-amilase e, consequentemente, menor velocidade de germinação. A $\alpha$-amilase desempenha um papel fundamental na hidrólise do amido, contribuindo para a germinação (KATO-NOGUCHI 2008). No entanto, o efeito inibitório do eugenol pode ser aliviado ao decorrer do tempo, devido a sua volatilidade.

Em sementes de pimentão tratadas com $\mathrm{OE}$ de cravo, contendo $81 \%$ de eugenol em sua composição, apesar da primeira contagem de germina- ção ter sido baixa, após 14 dias, 95\% das sementes haviam germinado, não diferindo estatisticamente do controle (94\%) (NASCIMENTO et al. 2020). Esse resultado demonstra que possivelmente o eugenol leve a um "atraso" na germinação, não afetando, contudo, o vigor das plântulas. De modo semelhante, sementes de arroz e trigo embebidas em soluções de eugenol ( $2 \mathrm{~g} / \mathrm{L}$ e 0,4 g/L, respectivamente) apresentaram menor velocidade de germinação (DARABI et al. 2011; HU et al. 2017).

A imersão de sementes de feijão-fava em água destilada contendo OE de cravo $(2 \mathrm{~mL} / \mathrm{L})$ mais emulsificante, afetou negativamente a primeira contagem e índice de velocidade de emergência. No entanto, resultados positivos foram obtidos com menores concentrações (GOMES et al. 2016).

\section{ENCAPSULAMENTO DE ÓLEOS ESSENCIAIS}

Características inerentes à composição dos OEs, como alta volatilidade, rápida degradação e baixa miscibilidade em soluções aquosas, têm impossibilitado resultados satisfatórios com sua aplicação a campo (CADENA et al. 2018). Logo, novas formulações são necessárias para melhorar sua estabilidade.

A nanoencapsulação é uma das mais interessantes inovações agrícolas que pode ser usada como ferramenta para criação de biodefensivos, com liberação controlada de substâncias biologicamente ativas, como os OEs (NURUZZAMAN et al. 2016; WORRALL et al. 2018).

No caso dos OEs, o encapsulamento em nanopartículas, como nanopartículas de sílica mesoporosa (MSNPs), pode evitar sua volatilização, melhorando sua estabilidade a longo prazo (BERNARDOS et al. 2015; CADENA et al. 2018; CHAN et al. 2017). Os MSNPs, também conhecidos como sílica, são compostos de dióxido de silício ( $\mathrm{SiO} 2)$ que se decompõe naturalmente e são inofensivos ao meio ambiente (DIACONU et al. 2010; SLOWING et al. 2008). A sílica é decomposta em subprodutos do ácido silícico, podendo atuar ainda como bioestimulantes para o crescimento da planta, melhorando sua resistência a pragas e doenças (DIACONU et al. 2010; REYNOLDS et al. 2016).

Cadena et al. (2018) verificaram que o encapsulamento de cinamaldeído, composto majoritário do OE de canela, com MSNPs revestidos com alginato de sódio, prolongou a atividade antimicrobiana do composto, protegendo-o da degradação e eva- 
poração. Além disso, a incorporação de compostos hidrofóbicos e voláteis em uma formulação como essa requer doses baixíssimas, evitando o uso de doses maiores que poderiam afetar negativamente a germinação e desenvolvimento da planta. A liberação lenta e controlada resultou em maior eficácia no controle de Pseudomonas syringae pv. pisi em sementes de ervilha (CADENA et al. 2018).

Sattary et al. (2020) relataram $74,4 \%$ e $71,27 \%$ de controle do mal-do-pé (Gaeumannomyces graminis var. tritici) em sementes de trigo tratadas com os OEs de capim-limão e cravo, respectivamente. Para tanto, as sementes foram imersas em água destilada esterilizada contendo os OEs encapsulados em MSNPs, por 30 minutos. Os autores constataram ainda que, o tratamento das sementes com os dois OEs mais o revestimento com alginato, elevou a taxa de controle da doença para $84 \%$. Óleos essenciais de capim limão e cravo, puros, apresentaram uma taxa de controle inferior $(57,44 \%$ e $48,93 \%$, respectivamente).

\section{CONSIDERAÇÕES FINAIS}

Conforme abordado, os óleos essenciais têm demonstrado atividade antimicrobiana contra ampla gama de fitopatógenos, porém, os estudos sobre seu emprego em tratamento de sementes ainda são escassos. Considerando o interesse cada vez maior na questão de sustentabilidade e na produção orgânica, com alimentos de melhor qualidade, é inegável que essa área de pesquisa apresenta grande potencial de medida alternativa a compor o manejo integrado de doenças de plantas.

Se comparado aos defensivos tradicionais, os custos ainda são elevados, em parte devido à carência de fornecedores, mas com a maior difusão desses produtos e maior envolvimento das empresas, esse gargalo pode ser sanado. $\mathrm{O}$ uso do óleo de neem no controle de doenças e pragas, através da pulverização foliar, por exemplo, já está amplamente disseminado, com inúmeros produtos registrados. No entanto, produtos derivados de plantas têm muito mais a oferecer a nossa agricultura e, a área da patologia de sementes é uma dessas ainda a ser explorada. Uma vez preenchida a lacuna entre pesquisadores e empresas, com a transferência de todo conhecimento existente, os agricultores poderão se beneficiar com mais um produto no manejo alternativo de doenças transmitidas por sementes.

\section{REFERÊNCIAS}

ABDEL-KADER M, EL-MOUGY N, LASHIN S (2011). Essential oils and Trichoderma harzianum as an integrated control measure against faba bean root rot pathogens. Journal of Plant Protection Research 5: 306-313. (https://doi.org/10.2478/ v10045-011-0050-8).

ABDIN MZ, AHMAD MM, JAVED S (2010). Advances in molecular detection of Aspergillus: an update. Archives of Microbiology 192: 409-425. (https://doi.org/10.1007/s00203-010-0563-y).

AJAYI OE, APPEL AG, FADAMIRO HY (2014). Phytotoxicity of some essential oil components to cowpea (Vigna unguiculata (L.) Walp.) seeds. International Journal of Plant Biology \& Research 2: 1024.

ARAUJO NETO, AC, ARAÚJO PC, SOUZA WCO, MEDEIROS JGF, AGUIAR AVM (2012). Óleo essencial de anis na incidência e controle de patógenos em sementes de erva-doce, Foeniculum vulgare mill. Revista Verde de Agroecologia e Desenvolvimento Sustentável 7: 31.

ARSHAD Z, HANIF MA, QUADRI RWK, KHAN MM (2014). Role of essential oils in plant diseases protection: a review. International Journal of Chemical and Biochemical Sciences 6: 11-17.

BANKOLE SA, JODA AO, ASHIDI JS (2005). The use of powder and essential oil of Cymbopogon citratus against mould deterioration and aflatoxin contamination of "egusi" melon seeds. Journal of Basic Microbiology: An International Journal on Biochemistry, Physiology, Genetics, Morphology, and Ecology of Microorganisms 45: 20-30. (https://doi.org/10.1002/jobm.200410435).

BARD $M$, ALBRECHT MR, GUPTA N, GUYNN CJ, STILLWELL, W (1988). Geraniol interferes with membrane functions in strains of Candida and Saccharomyces. Lipids 23: 534-538. (https:// doi.org/10.1007/BF02535593).

BASAK S, GUHA P (2017). Betel leaf (Piper betle L.) essential oil microemulsion characterization and antifungal activity on growth, and apparent lag time of Aspergillus flavus in tomato paste. LWT 75 :616-623. (https://doi.org/10.1016/j. Iwt.2016.10.021).

BERNARDOS A, MARINA T, ZACEK P, PÉREZ-ESTEVE E, MARTÍNEZ-MANEZ R, LHOTKA M, KOURIMSKA L, PULKRABEK J, KLOUCEK P (2015). Antifungal effect of essential oil components 
against Aspergillus when loaded into silica mesoporous supports. Journal of the Science of Food and Agriculture 95: 2824-2831. (https:// doi.org/10.1002/jsfa.7022).

CADENA M,B PRESTON, GM, VAN DER HOORN, RAL, FLANAGAN NA, TOWNLEY HE, THOMPSONIP (2018). Enhancing cinnamon essential oil activity by nanoparticle encapsulation to control seed pathogens. Industrial Crops and Products 124: 755-764. (https://doi.org/10.1016/j.indcrop.2018.08.043).

CHAN AC, CADENA MB, TOWNLEY HE, FRICKER MD, THOMPSON IP (2017). Effective delivery of volatile biocides employing mesoporous silicates for treating biofilms. Journal of The Royal Society Interface 14. (https://doi.org/10.1098/ rsif.2016.0650).

CHECHI A, GHISSI-MAZETTI VC, ZUCHELLI E, DEUNER CC, FORCELINI CA, BOLLER W (2020). In vivo sensitivity of Phakopsora pachyrhizi to fungicides. Ciência Rural 50. (https://doi.org/10.1590/0103-8478cr20190593).

CHENG SS, LIU JY, CHANG EH, CHANG ST (2008). Antifungal activity of cinnamaldehyde and eugenol congeners against wood-rot fungi. Bioresource technology 99: 5145-5149. (https://doi. org/10.1016/j.biortech.2007.09.013).

CHOU CH (1999). Roles of allelopathy in plant biodiversity and sustainable agriculture. CriticalReviews in Plant Sciences 18: 609-636. (https:// doi.org/10.1080/07352689991309414).

COSTA ART, AMARAL MFZJ, MARTINS PM, PAULA JAM, FIUZA TS, TRESVENZOL LMF, PAULA JR, BARA MTF (2011). Ação do óleo essencial de Syzygium aromaticum (L.) Merr. \& LM Perry sobre as hifas de alguns fungos fitopatogênicos. Revista Brasileira de Plantas Medicinais 13: 240 245.

COX SD, GUSTAFSON JE, MANN CM, MARKHAM JL, LIEW YC, HARTLAND RP, BELL HC, WARMINGTON JR, WYLLIE SG (1998). Tea tree oil causes $\mathrm{K}+$ leakage and inhibits respiration in Escherichia coli. Letters in Applied Microbiology 26: 355-358. (https://doi.org/10.1046/j. 1472-765x.1998.00348.x).

COX SD, MANN CM, MARKHAM JL, BELL HC, GUSTAFSON JE, WARMINGTON JR, WYLLIE SG (2000). The mode of antimicrobial action of the essential oil of Melaleuca alternifolia (tea tree oil). Journal of Applied Microbiology 88: 170-175. (https://doi.org/10.1046/j. 1365-2672.2000.00943.x).

DARABI HR, MOHANDESSI S, BALAVAR $Y$, MOGHADDAM MM, AGHAPOOR K, MOHSENZADEH F, NOURINIA AA (2011). Clove bud oil: an efficient, economical and widely available oil for the inhibition of wheat seed germination. Environmental Chemistry Letters 9: 519-524. (https://doi.org/10.1007/s10311-011-0312-8.).

DIACONU M, TACHE A, EREMIA SAV, GATEA F, LITESCU SC, RADU GL (2010). Structural characterization of chitosan coated silicon nanoparticles-a FT-IR approach. UPB Scientific Bulletin 72.

DIAS LRC, SANTOS ARB, PAZ FILHO ER, SILVA PHS, ATHAYDE SOBRINHO C (2019). Óleo essencial de Lippia sidoides Cham (alecrim-pimenta) no controle de Macrophomina phaseolina em feijão-caupi. Embrapa Meio Norte.

DOMENE MP, GLÓRIA EM, BIAGI JD, BENEDETTI BC, MARTINS L (2016). Efeito do tratamento com óleos essenciais sobre a qualidade fisiológica e sanitária das sementes de milho (Zea mays). Arquivos do Instituto Biológico 83: 1-6. (https:// doi.org/10.1590/1808-1657000072014).

DUKE SO, DAYAN FE, RIMANDO AM, SCHRADER KK, ALIOTTA G, OLIVA A, ROMAGNI JG (2002). Chemicals from nature for weed management. Weed Science 50: 138-151. (https://doi.org/10.1614/0043-1745(2002)050[0138:IPCFNF]2.0.CO;2).

EL-MOUGY NS, ABDEL-KADER MM, ALY MDE, LASHIN SM (2012) Application of fungicides alternatives as seed treatment for controlling root rot of some vegetables in pot experiments. Advances in Life Sciences, 2:57-64.

ERYIGIT T, YILDIRIM B, EKICI E, ÇIRKA M (2017). Chemical composition, antimicrobial and antioxidant properties of Schinus molle L. essential Oil from Turkey. Journal of Essential Oil Bearing Plants 20: 570-577. (https://doi.org/10.1080/09 72060X.2017.1304286).

FELIPE LO, BICAS JL (2017). Terpenos: compostos majoritários de óleos essenciais. Química e Sociedade 39: 120-130. (http://dx.doi. org/10.21577/0104-8899.20160068).

FRAENKEL GS (1959). The raison d'etre of secondary plant substances. Science 1466-1470. (https://doi.org/10.1126/science.129.3361.1466). 
GHINI R, KIMATI H (2000). Resistência de fungos a fungicidas. Jaguariúna, SP. Embrapa Meio Ambiente.

GOMES RSS, NUNES MC, NASCIMENTO LC, SOUZA JO, PORCINO MM (2016). Eficiência de óleos essenciais na qualidade sanitária e fisiológica em sementes de feijão-fava (Phaseolus lunatus L.). Revista Brasileira de Plantas Medicinais 18:279-287. (https://doi.org/10.1590/ 1983-084X/15_117).

HAMMER KA, CARSON CF (2011). Antibacterial and antifungal activities of essential oils. In: Thormar H (Ed.) Lipids and Essential Oils as Antimicrobial Agents. Nova Jersey: John Wiley \& Sons. pp. 255-306.

HILLEN T, SCHWAN-ESTRADA KRF, MESQUINI RM, CRUZ MES, STANGARLIN JR, NOZAKI M (2012). Atividade antimicrobiana de óleos essenciais no controle de alguns fitopatógenos fúngicos in vitro e no tratamento de sementes. Revista Brasileira de Plantas Medicinais 14: 439-445. (https://doi.org/10.1590/S1516057220120003000030).

HU Q, LIN C, GUAN Y, SHETEIWY MS, HU W, HU J (2017). Inhibitory effect of eugenol on seed germination and pre-harvest sprouting of hybrid rice (Oryza sativa L.). Scientific Reports 7: 19. (https://doi.org/10.1038/s41598-017-04104-x).

ISMAN MB (2000). Plant essential oils for pest and disease management. Crop Protection 19: 603608. (https://doi.org/10.1016/S0261-2194(00)00079-X).

ISMAN MB, MACHIAL CM (2006). Pesticides based on plant essential oils: from traditional practice to commercialization. Advances in Phytomedicine 3: 29-44. (https://doi.org/10.1016/S1572557X(06)03002-9).

KATO-NOGUCHI H (2008). Effects of four benzoxazinoids on gibberellin-induced $\alpha$-amylase activity in barley seeds. Journal of Plant Physiology 165: 1889-1894. (https://doi.org/10.1016/j.jplph.2008.04.006).

KAYODE RMO, AFOLAYAN AJ (2015). Cytotoxicity and effect of extraction methods on the chemical composition of essential oils of Moringa oleifera seeds. Journal of Zhejiang University-SCIENCE B 16: 680-689. (https://doi.org/10.1631/jzus. B1400303).

KUMAR N (2016). Preservative potential of cumin essential oil for Pisum sativum L. during storage. Journal of Plant Protection Research 56: 203210. (https://doi.org/10.1515/jppr-2016-0027). LANINI WT (2010). Organic herbicides-do they work. California Weed Science Society Journal 6: 1-3.

LI Y, WANG Y, KONG W, YANG S, LUO J, YANG M (2020). Illicium verum essential oil, a potential natural fumigant in preservation of lotus seeds from fungal contamination. Food and Chemical Toxicology 141: 111347. (https://doi.org/10.1016/j.fct.2020.111347).

LO CANTORE P, SHANMUGAIAH V, IACOBELLIS NS (2009). Antibacterial activity of essential oil components and their potential use in seed disinfection. Journal of Agricultural and Food Chemistry 57: 9454-9461. (https://doi. org/10.1021/jf902333g).

LOBATO AKS, SANTOS DGC, OLIVEIRA FC, GOUVEA DDS, TORRES GIOS, LIMA JÚNIOR JA, OLIVEIRA NETO CF, SILVA MHL (2007). Ação do óleo essencial de Piper aduncum L . utilizado como fungicida natural no tratamento de sementes de Vigna unguiculata ( L .) Walp. Revista Brasileira de Biociências 5: 915-917.

MANSO S, CACHO-NERIN F, BECERRIL R, NERÍN C (2013). Combined analytical and microbiological tools to study the effect on Aspergillus flavus of cinnamon essential oil contained in food packaging. Food Control 30: 370-378. (https://doi.org/10.1016/j.foodcont.2012.07.018).

MATA MF, ARAUJO E, NASCIMENTO LC, SOUZA AEF, VIANA S (2009). Incidência e controle alternativo de patógenos em sementes de mandacaru (Cereus jamacaru DC, Cactaceae). Revista Brasileira de Biociências 7: 327-334.

MBEGA E, MABAGALA RB, MORTENSEN CN, EDNAR W (2012). Evaluation of essential oils as seed treatment for the control of Xanthomonas spp. associated with the bacterial leaf spot of tomato in Tanzania. Journal of Plant Pathology 273-281. (http://dx.doi.org/10.4454/JPP. FA.2012.024).

MENEZES FILHO ACP, SOUSA WC, CASTRO CFD (2020a). Composição química dos óleos essenciais de schinus molle e atividade antifúngica frente à Sclerotinia sclerotiorum. Colloquium Agrariae 16: 115-123.

MENEZES FILHO ACP, SOUSA WC, CASTRO CFD 
(2020b). Composição química, físico-química e atividade antifúngica dos óleos essenciais da flor e do fruto de Myrcia guianensis (Aubl.) DC. Revista Principia 52: 92-104. (https://doi. org/10.18265/1517-0306a2020v1n52p92-104).

MISRA G, PAVLOSTATHIS SG (1997). Biodegradation kinetics of monoterpenes in liquid and soil slurry systems. Applied Microbiology and Biotechnology 47: 572-577. (https://doi. org/10.1007/s002530050975).

MONTES-BELMONT R, CARVAJAL M (1998). Control of Aspergillus flavus in maize with plant essential oils and their components. Journal of Food Protection 61: 616-619. (https://doi.org/10.4315/0362-028x-61.5.616).

MORAIS, LAS (2009a) Óleos essenciais no controle fitossanitário. In: Bettiol W, Morandi MAB(Eds) Biocontrole de doenças de plantas: uso e perspectivas. Jaguariúna: Embrapa. pp.139 152.

MORAIS LAS (2009b). Influência dos fatores abióticos na composição química dos óleos essenciais. Horticultura Brasileira 27: 4050-4063.

NASCIMENTO DM, SANTOS PL, RIBEIRO-JUNIOR MR, SARTORI MMP, KRONKA AZ (2020). Essential oils control anthracnose in pepper seeds. Research, Society and Development 9. (https:// doi.org/10.33448/rsd-v9i10.9028).

NASCIMENTO, DM, SANTOS PL, KRONKA AZ (2019). Essential oils inhibit Colletotrichum gloeosporioides spore germination. Summa Phytopathologica 45: 432-433. (https://doi. org/10.1590/0100-5405/207645).

Nerilo SM, Romoli JCZ, Nakasugi LP, Zampieri NS, Mossini SAG, Rocha GHO, Gloria EM, Abreu FiIho BA, Machinski Jr M (2020). Antifungal activity and inhibition of aflatoxins production by Zingiber officinale Roscoe essential oil against Aspergillus flavus in stored maize grains. Ciência Rural 50: 1-10. (https://doi.org/10.1590/ 0103-8478cr20190779).

NGUEFACK J, LETH V, DONGMO JBL, TORP J, ZOLLO PHA, NYASSE S (2008). Use of three essential oils as seed treatments against seed-borne fungi of rice (Oryza sativa L.). American-Eurasian Journal of Agricultural and Environmental Science 4: 554-560.

NURUZZAMAN MD, RAHMAN MM, LIU Y, NAIDU R (2016). Nanoencapsulation, nano-guard for pesticides: a new window for safe applica- tion. Journal of Agricultural and Food Chemistry 64: 1447-1483. (https://doi.org/10.1021/acs. jafc.5b05214).

OHNO T, KITA M, YAMASKA Y, IMAMURA S, YAMAMOTO T, MITSUFUJI S, KODAMA T, KASHIMA K, IMANISHI J (2003). Antimicrobial activity of essential oils against Helicobacter pylori. Helicobacter 8: 207-215. (https://doi.org/10.1046/ j.1523-5378.2003.00146.x).

ORZALI L, LUISON D, RICCIONI L (2014). Utilizzo di principi attivi di origine naturale per la concia delle sementi e per il controllo delle malattie trasmesse per seme. Dal. SEME 9: 4648.

PERAICA M, RADIC B, LUCIC A, PAVLOVIC M (1999). Toxic effects of mycotoxins in humans. Bulletin of the World Health Organization 77: 754.

RANA IS, RANA AS, RAJAK RC (2011). Evaluation of antifungal activity in essential oil of the Syzygium aromaticum (L.) by extraction, purification and analysis of its main component eugenol. Brazilian Journal of Microbiology 42: 12691277. (10.1590/S1517-83822011000400004).

RAUT JS, KARUPPAYIL SM (2014). A status review on the medicinal properties of essential oils. Industrial Crops and Products 62: 250-264. (https://doi.org/10.1016/j.indcrop.2014.05.055).

REYNOLDS OL, PADULA MP, ZENG R, GURR GM (2016). Silicon: potential to promote direct and indirect effects on plant defense against arthropod pests in agriculture. Frontiers in Plant Science 7: 744. (https://doi.org/10.3389/ fpls.2016.00744).

RIBEIRO SM, BONILLA OH, LUCENA EMP (2018). Influência da sazonalidade e do ciclo circadiano no rendimento e composição química dos óleos essenciais de Croton spp. da Caatinga. Iheringia. Série Botânica 73: 31-38.

RICCIONI L, ORZALI L, ROMANI M, ANNICCHIARICO P, PECETTI L (2019). Organic seed treatments with essential oils to control ascochyta blight in pea. European Journal of Plant Pathology 155: 831-840. (https://doi.org/10.1007/ s10658-019-01815-x).

RODRIGUES EA, SCHWAN-ESTRADA KRF, STANGARLIN JR, SCAPIM CA, FIORI-TUTIDA ACG (2006). Potencial da planta medicinal Ocimum gratissimum no controle de Bipolaris sorokiniana em sementes de trigo. Acta Scientiarum Agro- 
nomy 28: 213-220. (https://doi.org/10.4025/ actasciagron.v28i2.1071).

ROLLI E, MARIESCHI M, MAIETTI S, SACCHETTI G, BRUNI R (2014). Comparative phytotoxicity of 25 essential oils on pre-and post-emergence development of Solanum lycopersicum L.: A multivariate approach. Industrial Crops and Products 60: 280-290. (https://doi.org/10.1016/j. indcrop.2014.06.021).

SANTOS, PL (2018). Manejo de Macrophomina phaseolina (tassi) goid. em sementes de feijoeiro (Phaseolus vulgaris I.) com óleos essenciais e antagonistas. Tese, Universidade Estadual Paulista Júlio de Mesquita Filho. Botucatu, SP, Brasil.

SATTARY M, AMINI J, HALLAJ R (2020). Antifungal activity of the lemongrass and clove oil encapsulated in mesoporous silica nanoparticles against wheat's take-all disease. Pesticide Biochemistry and Physiology 170: 104696. (https://doi.org/10.1016/j.pestbp.2020.104696).

SHERALD JL, RAGSDALE NN, SISLER HD (1973). Similarities between the systemic fungicides triforine and triarimol. Pesticide Science 4: 719727. (https://doi.org/10.1002/ps.2780040514).

SHENGE KC (2006). Bacterial speck and spot diseases of tomato in Tanzania: pathogen characterization, epidemiology and management options. Ph.D. Thesis. Sokoine University of Agriculture, Morogoro, Tanzania.

SIDDIQUI SA, ISLAM R, JAMAL AHM, PARVIN T, RAHMAN A (2017). Chemical composition and antifungal properties of the essential oil and various extracts of Mikania scandens (L.) Willd. Arabian Journal of Chemistry 10: S2170-S2174. (https://doi.org/10.1016/j.arabjc.2013.07.050).

SILVA AC, SOUZA PE, MACHADO JC, SILVA BM, PINTO JEBP (2012). Effectiveness of essential oilsin the treatment of Colletotrichum truncatum-infected soybean seeds. Tropical Plant Pathology 37: 305-313. (https://doi.org/10.1590/ S1982-56762012000500001).

SILVA EAJ, SILVA VP, ALVES CCF, ALVES JM, SOUCHIE EL, BARBOSA LCA (2018). Chemical composition of the essential oil of Psidium guajava leaves and its toxicity against Sclerotinia sclerotiorum. Semina: Ciências Agrárias 39: 865-874. (https://doi.org/10.5433/1679-0359.2018v39n2p865).

SINDHU S, CHEMPAKAM B, LEELA NK, BHAI RS
(2011). Chemoprevention by essential oil of turmeric leaves (Curcuma longa L.) on the growth of Aspergillus flavus and aflatoxin production. Food and Chemical Toxicology 49: 1188-1192. (https://doi.org/10.1016/j.fct.2011.02.014).

SLOWING II, VIVERO-ESCOTO JL, WU CW, LIN VSY (2008). Mesoporous silica nanoparticles as controlled release drug delivery and gene transfection carriers. Advanced Drug Delivery Reviews 60: 1278-1288. (https://doi.org/10.1016/j. addr.2008.03.012).

SOMDA I, LETH V, SEREME P (2007). Antifungal effect of Cymbopogon citratus, Eucalyptus camaldulensis and Azadirachta indica oil extracts on sorghum seed-borne fungi. Asian Journal of Plant Sciences 2: 40-47.

STAMP N (2003). Out of the quagmire of plant defense hypotheses. The Quarterly Review of Biology 78: 23-55. (https://doi.org/10.1086/367580.).

STURCHIO E, DONNARUMMA L, ANNESI T, MILANO F, CASORRI L, MASCIARELLI E, ZANELLATO M, MECONI C, BOCCIA P (2014). Essential oils: an alternative approach to management of powdery Mildew diseases. Phytopathologia Mediterranea 385-395. (https://doi.org/10.14601/ Phytopathol_Mediterr-13607).

SUZUKI K, KATO T, TAKAHASHI J, KAMOSHITA K (1984). Mode of action of methyl N-(3,5 dichlorophenyl)-carbamate in the benzimidazoleresistant isolate of Botrytis cinerea. Journal of Pesticide Science 9: 497-501.

SWAMY MK, AKHTAR MS, SINNIAH UR (2016). Antimicrobial properties of plant essential oils against human pathogens and their mode of action: an updated review. Evidence-Based Complementary and Alternative Medicine. (https:// doi.org/10.1155/2016/3012462.).

SZABÓ MA, VARGA GZ, HOHMANN J, SCHULZ Z, SZEGEDI E, AMARAL L, MOLNÁR J (2010). Inhibition of quorum-sensing signals by essential oils. Phytotherapy Research 24: 782-786. (https://doi.org/10.1002/ptr.3010.)

TAIZ L, ZEIGER E (2013). Fisiologia vegetal. Porto Alegre: Artmed.

TASSOU C, KOUTSOUMANIS K, NYCHAS GJ (2000). Inhibition of Salmonella enteritidis and Staphylococcus aureus in nutrient broth by mint essential oil. Food Research International 33: 3-4. (https://doi.org/10.1016/S0963-9969(00)00047-8) 
VAN DER WOLF JM, BIRNBAUM Y, VAN DER ZOUWEN PS, GROOT SPC (2008). Disinfection of vegetable seed by treatment with essential oils, organic acids and plant extracts. Seed Science and Technology 36: 76-88. (https://doi. org/10.15258/sst.2008.36.1.08).

VYVYAN JR (2002). Allelochemicals as leads for new herbicides and agrochemicals. Tetrahedron 58: 1631-1646. (https://doi.org/10.1016/ S0040-4020(02)00052-2).

WALSH SE, MAILLARD JY, RUSSEL AD, CATRENICH CE, CHARBONNEAU DL, BARTOLO RG (2003). Activity and mechanisms of action of selected biocidal agents on Gram-positive andnegative bacteria. Journal of Applied Microbiology 94: 240-247. (https://doi.org/10.1046/j. 1365-2672.2003.01825.x.).

WANG C, ZHANG J, CHEN H, FAN Y, SHI Z (2010). Antifungal activity of eugenol against Bo- trytis cinerea. Tropical Plant Pathology 35: 137-143. (https://doi.org/10.1590/S198256762010000300001).

WORRAL EA, HAMID A, MODY KT, MITTER N, PAPPU HR (2018). Nanotechnology for plant disease management. Agronomy 8: 285. (https://doi. org/10.3390/agronomy8120285).

YOUNG, SL (2004). Natural product herbicides for control of annual vegetation along roadsides. Weed Technology 18: 580-587.

ZUZARTE M, SALGUEIRO L (2015). Essential oils chemistry. In: Sousa DP (Ed) Bioactive essential oils and cancer. Springer. pp.19-61.

ZYGADLO JA, GROW NR (1995). Comparative study of the antifungal activity of essential oils from aromatic plants growing wild in the central region of Argentina. Flavour and Fragrance Journal 10: 113-118. (https://doi.org/10.1002/ ffj.2730100210). 